\title{
Gestational trophoblastic neoplasm
}

INSERM

\section{Source}

INSERM. (1999). Orphanet: an online rare disease and orphan drug data base. Gestational trophoblastic neoplasm. ORPHA:59305

Gestational trophoblastic tumors (GTT) are malignant forms of gestational trophoblastic disease. The tumor always follows pregnancy, most often molar pregnancy

(hydatidiform mole; see this term). Four histological subtypes have been described: invasive mole, gestational choriocarcinoma, placental site trophoblastic tumor and epithelioid trophoblastic tumor (see these terms). 